\title{
DEFINIÇÃO DE ÁREAS PRIORITÁRIAS PARA A RECUPERAÇÃO FLORESTAL EM BACIAS HIDROGRÁFICAS A PARTIR DE ANÁLISE MỦLTICRITÉRIO
}

\author{
Renata Cristina Mafra \\ Universidade do Oeste Paulista \\ Programa de Pós-Graduação em Meio Ambiente e Desenvolvimento Regional \\ renatacmafra@outlook.com \\ Lucas Prado Osco \\ Universidade do Oeste Paulista \\ Faculdade de Engenharia e Arquitetura. Rodovia Raposo Tavares \\ pradoosco@gmail.com \\ Marcelo Rodrigo Alves \\ Universidade do Oeste Paulista \\ Programa de Pós-Graduação em Meio Ambiente e Desenvolvimento Regional \\ marceloalves@unoeste.br \\ Ana Paula Marques Ramos \\ Universidade do Oeste Paulista \\ Programa de Pós-Graduação em Meio Ambiente e Desenvolvimento Regional \\ anaramos@unoeste.br
}

\begin{abstract}
RESUMO
A recuperação florestal em bacias hidrográficas é essencial para aumentar a produção de água de boa qualidade para consumo. Contudo, essa prática exige um planejamento adequado que aponte áreas prioritárias para o investimento de recursos econômicos e humanos no reflorestamento. $\mathrm{O}$ objetivo deste estudo foi identificar as áreas prioritárias para a recuperação florestal em bacias hidrográficas por meio de Análise Multicritério em ambiente SIG. Para o estudo de caso adotamos a bacia hidrográfica do rio Pirapozinho, São Paulo. Como critérios relevantes para a determinação de áreas prioritárias, adotamos: rede de drenagem, distância de rodovias, distância de áreas urbanas, fragmentos de vegetação, e vulnerabilidade a erosão. Os pesos de cada critério foram obtidos a partir do Processo Analítico Hierárquico (AHP). Testamos dois métodos para criação do mapa síntese: CLP (Combinação Linear Ponderada) e operador Fuzzy Gamma. Como resultado obtivemos dois cenários; o primeiro com o método CLP onde priorizamos áreas com fragmentos de vegetação e alta densidade de drenagem, e o segundo com o método Gamma, que priorizou áreas vulneráveis da bacia. Concluímos que o modelo de integração proposto satisfaz a identificação de áreas para a recuperação de florestas em bacias hidrográficas, e que diferentes cenários podem ser construídos.
\end{abstract}

Palavras-chave: Inferência geográfica. Análise multicritério. Recuperação florestal. Áreas prioritárias. SIG.

\section{DEFINITION OF PRIORITY AREAS FOR FOREST RECOVERY IN HYDROGRAPHIC BASINS AND PARTIAL MULTICRITERY ANALYSIS}

\begin{abstract}
Forest recovery in river basins is essential to increase the production of good quality drinking water. However, this practice requires proper planning that points out priority areas for investing economic and human resources in reforestation. The objective of this study was to identify priority areas for forest recovery in watersheds by means of Multicriteria Analysis in GIS environment. For the case study we adopted the hydrographic basin of the Pirapozinho River, São Paulo. As relevant criteria for determining priority areas, we adopted: drainage network, distance from highways, distance from urban areas, fragments of vegetation, and vulnerability to erosion. The weights of each criterion were obtained from the
\end{abstract}


Hierarchical Analytical Process (AHP). We tested two methods for creating the synthesis map: PLC (Weighted Linear Combination) and Fuzzy Gamma operator. As a result we got two scenarios; the first with the PLC method where we prioritize areas with vegetation fragments and high drainage density, and the second with the Gamma method, which prioritized geotechnically vulnerable areas of the basin. We conclude that the proposed integration model satisfies the identification of areas for forest restoration in watersheds, and that different scenarios can be constructed.

Keywords: Geographical inference. Multicriteria analysis. Forest recovery. Priority areas. GIS.

\section{INTRODUÇÃO}

A supressão de áreas florestadas e a sua substituição por outras coberturas contribuem de forma significativa para os processos de desagregação, transporte e deposição de sedimentos nos canais de drenagem (SERPA et al., 2015; OUYANG et al., 2018; WU et al., 2018). As matas ciliares protegem a superfície do solo dos processos erosivos, assim como a vegetação ao redor de nascentes as protegem contra a compactação e o assoreamento (FILOSO et al., 2017; MAGNANI et al., 2018; SUN et al., 2018).

A ausência da vegetação pode causar mudanças na interação do ecossistema, especialmente na dispersão de populações e no isolamento reprodutivo (VALENTE et al., 2017). Isso pode causar problemas e áreas pequenas podem não oferecer alimento suficiente para manter populações viáveis, fazendo com que parte das espécies seja aos poucos extinta (BRANCALION et al., 2016).

Afim de garantir a conservação e a preservação da estrutura e processos da paisagem, o reflorestamento dirigido em bacias hidrográficas, notadamente por meio de ações de recuperação florestal em áreas prioritárias, tem sido apontado como um dos principais meios para aumentar a produção de água de boa qualidade e, portanto, um método efetivo e econômico no manejo de bacias. Exemplos de trabalhos nessa temática são realizados no México (Uribe et al., 2014), na China (Hu, et al.; 2017), na África (MONSTERT et al., 2018), e no Brasil Catelani et al. (2012), Sartori et al. (2012), Nossack et al. (2014), Vettorazzi et al. (2016), Valente et al. (2017), Mello et al. (2018), Almeida (2019).

No que se refere à espacialização dessas ações, o principal componente com a capacidade de integração e análise dos diferentes fatores que compõem a paisagem são os Sistema de Informação Geográfica (SIG), que tem facilitado o planejamento, a otimização e o sucesso das ações de conservação e de preservação florestal (KANGAS et al., 2000). Esse tipo de análise envolve um conjunto de alternativas de decisão, as quais são baseadas em vários critérios de avaliação (MALCZEWSKI, 2010).

Em uma abordagem multicritério, os critérios característicos de uma paisagem são representados por pesos diferentes, o que define a importância de cada um desses fatores no processo de tomada de decisão. Uma das técnicas mais tradicionais para desenvolver a análise multicritério é a proposta por Thomas Saaty em 1978 denominada de Processo Analítico Hierárquico (AHP - Analytic Hierarchy Process) (SAATY, 1990). O princípio é atribuir o peso aos critérios a partir de uma comparação pareada entre esses. Para tanto, Saaty propôs uma escala de 1 a 9, denominada de Escala Fundamental, sendo o valor 1 atribuído aos critérios de mesma importância relativa, e o valor 9 aos critérios de importância absoluta em relação aos demais (SAATY, 1990).

A partir da definição dos pesos dos critérios pelo AHP gera-se um mapa síntese pela Combinação Linear Ponderada desses critérios ou, menos tradicionalmente, pela Média Ponderada Ordenada dos critérios (VALENTE e VETTORAZZI, 2005; 2008; URIBE et al., 2014).

A análise multicriterial usando o AHP foi empregada em diferentes áreas atendendo a distintas aplicações, incluindo o diagnóstico ambiental de bacias hidrográficas, como em Ross (1994), Valente et al. (2008), Fushimi et al. (2013), Nossack et al. (2014), Uribe et al. (2014), Vettorazzi et al. (2016),

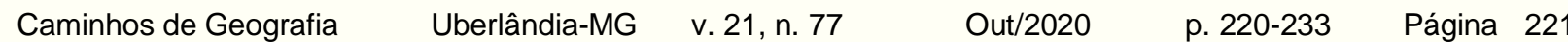


Gonçalves et al. (2016), Hu et al. (2017), Mello et al. (2018), Monstert et al. (2018), Rodrigues et al. (2018) e Fushimi et al. (2018). Considerando a literatura, observou-se a ausência de trabalhos testando métodos diferentes para a criação dos mapas síntese para áreas prioritárias à recuperação florestal, como CLP e operador Fuzzy Gamma.

Um importante método no processo de inferência geográfica é a lógica Fuzzy, que corresponde a uma extensão da lógica Booleana visando manipular o conceito de "verdade parcial", isto é, valores compreendidos entre "completamente verdadeiro" e "completamente falso" (CÂMARA et al., 2001). A lógica Fuzzy pode ser aplicada usando diferentes operadores para a combinação dos critérios na análise multicritério, sendo um deles o operador Gamma. Utilizar conjuntos Fuzzy tornam os conceitos imprecisos, encontrados no meio físico, mais próximos da realidade (GONÇALVES et al., 2016).

Uma região no estado de São Paulo com sérios problemas ambientais devido à forma de uso e ocupação de suas terras é o Pontal do Paranapanema. Essa área, de aproximadamente $19 \mathrm{mil} \mathrm{km} 2$, é considerada protagonista nas discussões acerca do processo de ocupação territorial e a participação do agronegócio canavieiro (ARANA, 2019). Segundo a Fundação Instituto de Terras do Estado de São Paulo (ITESP, 2013), a região possui um histórico de intensa ocupação territorial e, de uma forma geral, sem o devido planejamento ambiental que vise reduzir os impactos oriundos dessa ocupação.

O Pontal do Paranapanema é formado por 7 bacias hidrográficas, sendo uma delas a do rio Pirapozinho com $1000 \mathrm{~km} 2$. A atual forma de uso e ocupação da bacia do rio Pirapozinho tem contribuído de forma negativa para a interferência na qualidade de seus recursos hídricos (RODRIGUES et al., 2018). Essa bacia possui um déficit vegetativo muito alto, visto que apenas $6,5 \%$ do total da área possui vegetação. As áreas de pastagem e de culturas correspondem a 62,2\% e $26,1 \%$ respectivamente (RODRIGUES et al., 2018).

A característica de extensão territorial, disponibilidade de água em quantidade e qualidade dessa bacia indica a necessidade de recuperação da sua vegetação. Esse cenário evidencia a necessidade de novos estudos nessa temática voltado para a bacia do Rio Pirapozinho, considerando suas características e utilizando outros métodos, além do que está sendo abordado na literatura. Também é importante destacar a possibilidade do seu uso futuro para abastecimento público de água dos núcleos populacionais dentro ou próximos dessa bacia. Neste contexto, o objetivo deste estudo foi identificar as áreas prioritárias para a recuperação florestal em bacias hidrográficas por meio de Análise Multicritério em ambiente SIG.

\section{METODOLOGIA}

\section{Área de estudo}

A área de estudo é a bacia hidrográfica do rio Pirapozinho e possui uma área aproximada de 1.000 $\mathrm{km}^{2}$ (Figura 1). Está inserida na $22^{\mathrm{a}}$ Unidade de Gerenciamento de Recursos Hídricos do Pontal do Paranapanema (UGRHI-22) localizada no extremo oeste do estado de São Paulo. Uma característica importante da bacia do rio Pirapozinho é a sua potencialidade de atuar no abastecimento público dos núcleos populacionais mais próximos na região do Pontal do Paranapanema. 
Figura 1 - Área de estudo.

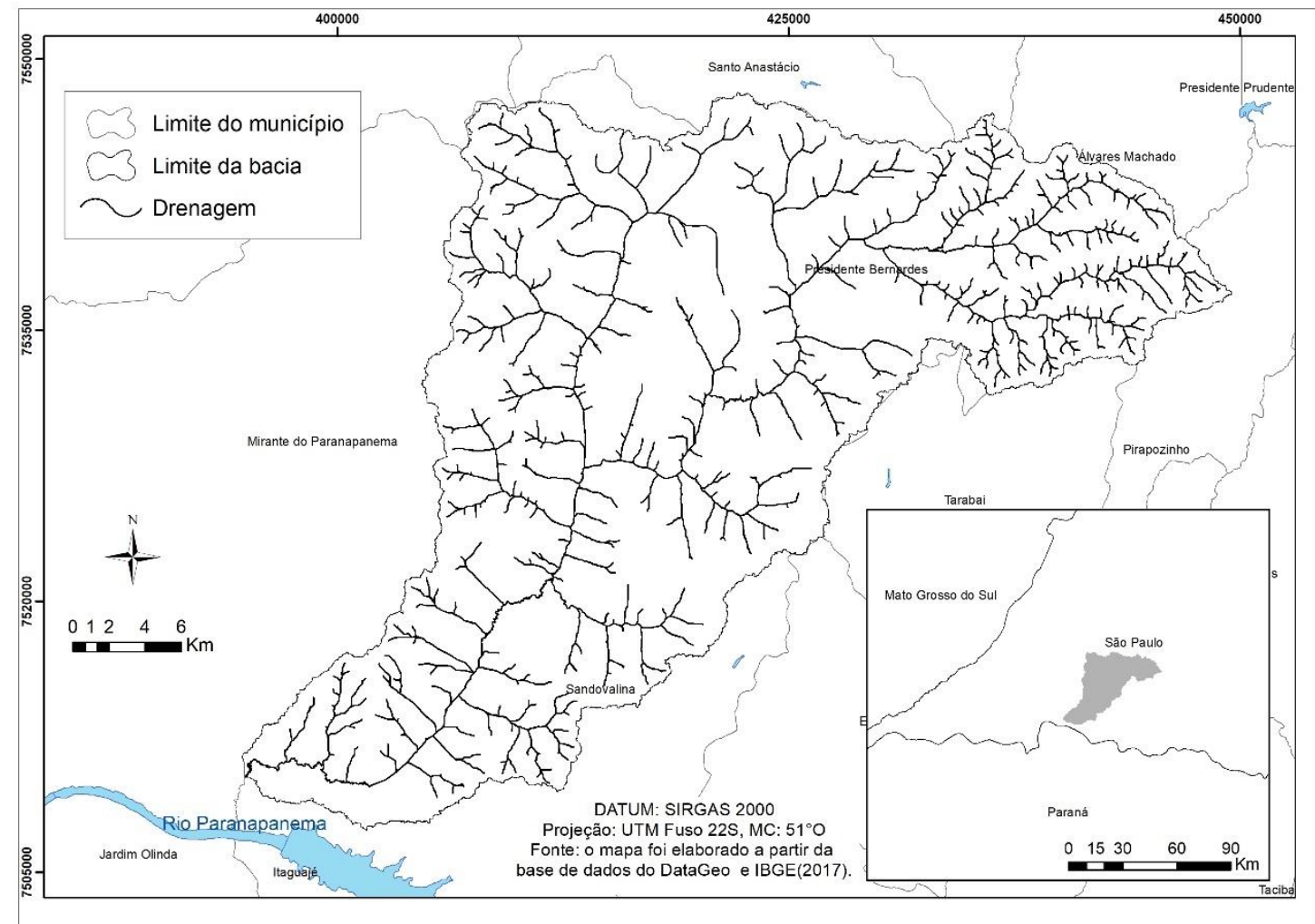

Fonte - Elaborado pelos autores.

\section{Organização dos dados cartográficos}

Os critérios utilizados para elaboração do mapa de áreas prioritárias para recuperação florestal foram estabelecidos de acordo com a literatura (VALENTE et al., 2005; VALENTE et al., 2008; CATELLANI et al., 2012; SARTORI et al., 2012; NOSSACK et al., 2014; VETTORAZZI et al., 2016; VALENTE et al., 2017; MELLO et al., 2018; BIANCHINI et al., 2019). Os critérios selecionados foram:

Vulnerabilidade à erosão: está relacionado com o nível de suscetibilidade a erosão que se encontra a bacia, de acordo com Mafra et al. (2020), levando em consideração 5 critérios. Para os critérios 'geomorfologia' e 'pedologia' foram utilizados os dados do Instituto de Pesquisa Tecnológica (IPT), de 1999, na escala 1:250.000. O critério 'uso e cobertura da terra' foram baixados na base de dados contínua do Instituto Brasileiro de Geografia e Estatística (IBGE) de 2017, escala 1:100.000.Para os critérios 'densidade de drenagem' e 'declividade' foram extraídos do MDS SRTM (NASA, 2020).

Distância de rodovias: a redução de ecossistema é causada direta ou indiretamente pelas rodovias. Refere-se à alteração da sua estrutura e composição, além da redução da área total de um ecossistema ou sua redução parcial, criando um mosaico de fragmentos (DINIZ et al., 1998; HARRIS, 1984; SOUTHWORTH et al., 2004; TURNER , 1989; VIANNA et al., 1997; REZZADORI et al., 2016).

Distância de áreas urbanas: a proximidade entre áreas urbanas e fragmentos florestais pode ser desfavorável pelas perturbações como caça, desmatamento e/ou incêndios (SARTORI et al., 2012). Para o sucesso de uma recuperação florestal é importante a priorização de áreas distantes dessas fontes de perturbação, uma vez que a qualidade da água está negativamente relacionada à urbanização (MELLO et al., 2018).

Fragmentos de vegetação: os córregos e as nascentes próximos a vegetação têm melhor qualidade de água do que áreas distantes desses fragmentos (PINTO et. al, 2012). As taxas de extinção também estão relacionadas aos fragmentos florestais, visto que fragmentos menores estão mais suscetíveis a perda maior de espécies do que fragmentos maiores. Fragmentos menores estão mais expostos a ações externas, seja ela pelos efeitos do uso e ocupação da terra ou outra atividade

\begin{tabular}{|c|c|}
\hline Caminhos de Geografia & Uberlândia-MG \\
\hline
\end{tabular}


antrópica (REZZADORI et al., 2016). Além disso, a biodiversidade não é afetada apenas pelo tamanho das manchas de floresta, mas também pela distância entre habitats vizinhos que podem desempenhar um papel importante na conectividade entre as espécies (POOR et al., 2019).

Rede de drenagem: a proximidade da rede hidrográfica tem sua importância, uma vez que a mata ciliar previne a erosão do solo e consequentemente mantém a qualidade da água (FILOSO et al., 2017; SUN et al., 2018).

Os mapas temáticos, representando cada critério foram organizados em um banco de dados geográfico no referencial geodésico adotado no Brasil, que é o SIRGAS 2000 (Sistema de Referência Geocêntrico para as Américas), e na projeção UTM (Universal Transverso de Mercator) fuso 22 Sul.

Para delimitar a bacia do rio Pirapozinho utilizamos a ferramenta Hidrology no software comercial ArcGIS 10.2, usando um Modelo Digital de Superfície, de $30 \mathrm{~m}$ de resolução espacial, coletado na missão SRTM (Shuttle Radar Topograpy Mission) da NASA (National Aeronautics and Space Administration) em 2000. A partir disso, foi usado o limite da área da bacia como máscara de recorte para os mapas temáticos de interesse.

Para os critérios "distância de rodovia", "distância de áreas urbanas" e "rede de drenagem" geramos mapas de distâncias por meio da ferramenta Euclidean distance do software ArcGIS 10.2. Elaboramos o critério "fragmento de vegetação" a partir dos dados do Mapeamento Anual da Cobertura e Uso do Solo no Brasil - MapBiomas (2018) que identifica o grau de cobertura vegetal presente na área de estudo. Classificamos os dados de acordo com o tamanho de seus fragmentos e separamos por classes (Tabela 1). Em seguida elaboramos mapas de distância de cada classe e definimos seus respectivos pesos. O maior fragmento recebeu o peso 1, e conforme encurtávamos as classes com os tamanhos dos fragmentos, os pesos foram diminuídos ao meio, respectivamente. Posteriormente aplicamos a álgebra de mapas, gerando o mapa síntese de fragmento de vegetação.

Tabela 1 - Tamanho dos fragmentos de vegetação e seus respectivos pesos.

\begin{tabular}{ccc}
\hline \multirow{2}{*}{ Classe } & Tamanho do fragmento & Pesos \\
& & \\
\hline $\mathbf{1}$ & Até 1 ha & 0,03125 \\
\hline $\mathbf{2}$ & $1-10$ ha & 0,0625 \\
\hline $\mathbf{3}$ & $10-50$ ha & 0,125 \\
\hline $\mathbf{4}$ & $50-150$ ha & 0,25 \\
\hline $\mathbf{6}$ & $150-300$ ha & 0,5 \\
\hline
\end{tabular}

Fonte - Elaborado pelos autores.

\section{Atribuição dos pesos usando o AHP}

A matriz de comparação (Tabela 2) foi elaborada para definir a importância dos critérios por meio de um processo de tomada de decisão conhecido como Análise Hierárquica Analítica - AHP (SAATY, 1990). Os critérios foram comparados (comparação pareada) tendo como referência a escala contínua de nove pontos (Figura 2) expressando a ordem de importância.

Figura 2 - Escala contínua para elaboração da matriz de comparação pareada.

\begin{tabular}{|c|c|c|c|c|c|c|c|c|}
\hline $1 / 9$ & $1 / 7$ & $1 / 5$ & $1 / 3$ & 1 & 3 & 5 & 7 & 9 \\
\hline Extremamente & $\begin{array}{c}\text { Muito } \\
\text { fortemente }\end{array}$ & Fortemente & Moderadamente & Igualmente & Moderadamente & Fortemente & $\begin{array}{c}\text { Muito } \\
\text { fortemente }\end{array}$ & Extremamente \\
\hline
\end{tabular}

Fonte - Eastman (2001). 
Tabela 2 - Matriz de pesos dos critérios definidos pelo AHP.

\begin{tabular}{|c|c|c|c|c|c|c|}
\hline Critérios & Vegetação & $\begin{array}{c}\text { Rede de } \\
\text { drenagem }\end{array}$ & $\begin{array}{l}\text { Vulnerabilidade } \\
\text { à erosão }\end{array}$ & $\begin{array}{c}\text { Distância } \\
\text { de } \\
\text { rodovias }\end{array}$ & $\begin{array}{l}\text { Distância } \\
\text { de áreas } \\
\text { urbanas }\end{array}$ & Pesos \\
\hline Vegetação & 1 & & & & & 0,494146 \\
\hline $\begin{array}{c}\text { Rede de } \\
\text { drenagem }\end{array}$ & $1 / 3$ & 1 & & & & 0,282236 \\
\hline $\begin{array}{l}\text { Vulnerabilidade } \\
\text { à erosão }\end{array}$ & $1 / 5$ & $1 / 3$ & 1 & & & 0,151756 \\
\hline $\begin{array}{l}\text { Distância de } \\
\text { rodovias }\end{array}$ & $1 / 9$ & $1 / 7$ & $1 / 5$ & 1 & & 0,035930 \\
\hline $\begin{array}{l}\text { Distância de } \\
\text { áreas urbanas }\end{array}$ & $1 / 9$ & $1 / 7$ & $1 / 5$ & 1 & 1 & 0,035930 \\
\hline
\end{tabular}

Fonte - Elaborado pelos autores.

\section{Inferência geográfica por diferentes modelos lógicos}

Antes da combinação dos critérios pela álgebra de mapas, padronizamos os dados em uma escala comum de 0 e 1 . Os critérios tiveram as relações de prioridade estabelecidas por meio da ferramenta Fuzzy Membership do software ArcGIS 10.2. Os critérios "distâncias de área urbana" e "distâncias de rodovias" foram ajustados com uma função linear crescente, ou seja, quanto mais distante maior a prioridade para reflorestar. Já os critérios "vulnerabilidade à erosão", "distância de drenagem" e "fragmentos de vegetação" foram ajustados com uma função linear decrescente, ou seja, quanto mais próximo, maior a prioridade de reflorestar.

Elaboramos o mapa de áreas prioritárias para recuperação florestal por diferentes modelos lógicos de inferência geográfica, como Combinação Linear Ponderada (CLP) juntamente com o método AHP. Esse método aplicado por meio da multiplicação de cada mapa de critérios (isto é, cada célula, ou pixel de cada mapa) pelo seu peso e, então, somados. O método foi proposto por Voogd (1983) e seu resultado é um mapa quantitativo expressando o grau de importância relativa por meio dos valores numéricos de saída (CÂMARA et al., 2001).

Outro tipo de combinação de dados é baseado na inferência Fuzzy, que utiliza métodos lógicos para combinar dados com um conjunto de critérios, portanto, também pode ser utilizado para se obter um mapa síntese de vários planos de informação. Dentre os operadores Fuzzy têm-se: produto algébrico; soma algébrica e; operador Gamma.

O produto algébrico possui a tendência de produzir valores muito pequenos devido a multiplicação de valores menores que 1, e seus valores de pertinência Fuzzy de saída é a multiplicação dos valores dos pixels dos mapas de entrada referente a cada critério. A soma algébrica representa um operador complementar ao produto algébrico que causa um aumento do valor de pertinência, ou seja, o resultado é sempre um valor maior ou igual ao mais valor de pertinência Fuzzy de entrada (CÂMARA et al., 2001).

O operador Gamma é definido pela multiplicação dos dois termos, um produto algébrico Fuzzy e uma soma algébrica Fuzzy. Este operador é expresso pela função:

$$
\mu=(\text { Soma algébrica Fuzzy })^{\vee} \times(\text { Produto algébrico Fuzzy })^{1-\vee}
$$

Pode-se variar a importância de cada termo (soma algébrica e produto algébrico Fuzzy) no operador Gamma. O grau de importância é definido por meio de atribuição de valores entre $(0,1)$ para o expoente " $\mathrm{Y}$ ". Adotamos três variações para o " $\mathrm{Y}$ ", sendo 0,4; 0,6 e 0,8 para identificar a melhor dispersão do conjunto de pontos de cada modelo. 


\section{RESULTADOS E DISCUSSÃO}

Para a definição de áreas prioritárias para reflorestamento na bacia em estudo elaboramos mapas de distância (Figura 3). Cada mapa foi feito a partir dos critérios utilizados, e diante da sua importância, teve função linear crescente ou decrescente. Os critérios "distância de rodovias" e "distância de áreas urbanas" tiveram uma função linear crescente, pois quanto mais distante dessas áreas, maior seria a prioridade para o reflorestamento. Uma justificativa para isso seria a interferência antrópica, uma vez que sua relação está diretamente ligada a perturbação e redução parcial ou total de fragmentos florestais.

Os critérios "distância de drenagem", "fragmentos de vegetação" tiveram uma função linear decrescente, pois quanto mais próxima estiver dessa área, maior a prioridade para reflorestar. A importância de priorizar essas áreas não está somente relacionada a qualidade da água, mas ao equilíbrio dos ecossistemas e conservação da biodiversidade em diferentes tamanhos de fragmentos florestais.

O critério "vulnerabilidade a erosão" também teve uma função linear decrescente, ou seja, quanto mais próximo a áreas vulneráveis, maior a prioridade para reflorestar. Esse critério foi baseado no estudo de Mafra et al. (2020), que testou mapas de vulnerabilidade à erosão para a bacia hidrográfica do rio Pirapozinho por diferentes modelos de inferência geográfica. Para a construção deste mapa houve a combinação de diferentes critérios e pesos. O critério que teve o maior peso foi a "declividade", e o segundo maior peso foi a "densidade de drenagem". Portanto, a cor verde escuro em alguns pontos da bacia no mapa de vulnerabilidade a erosão, demonstra uma densidade de drenagem e declividade menor que as outras regiões, desse modo uma menor prioridade de reflorestamento. Nas regiões mais claras existe uma concentração maior de afluentes e declividades maiores, portanto uma maior prioridade de recuperação florestal.

Figura 3 - Mapa de distância para definição das áreas prioritárias para reflorestamento na bacia hidrográfica do rio Pirapozinho. (A) Distância entre fragmentos de vegetação; (B) Distância de rodovias; (C) Distância de áreas urbanas; (D) Distância de drenagem; (E) Distância de áreas vulneráveis a erosão.
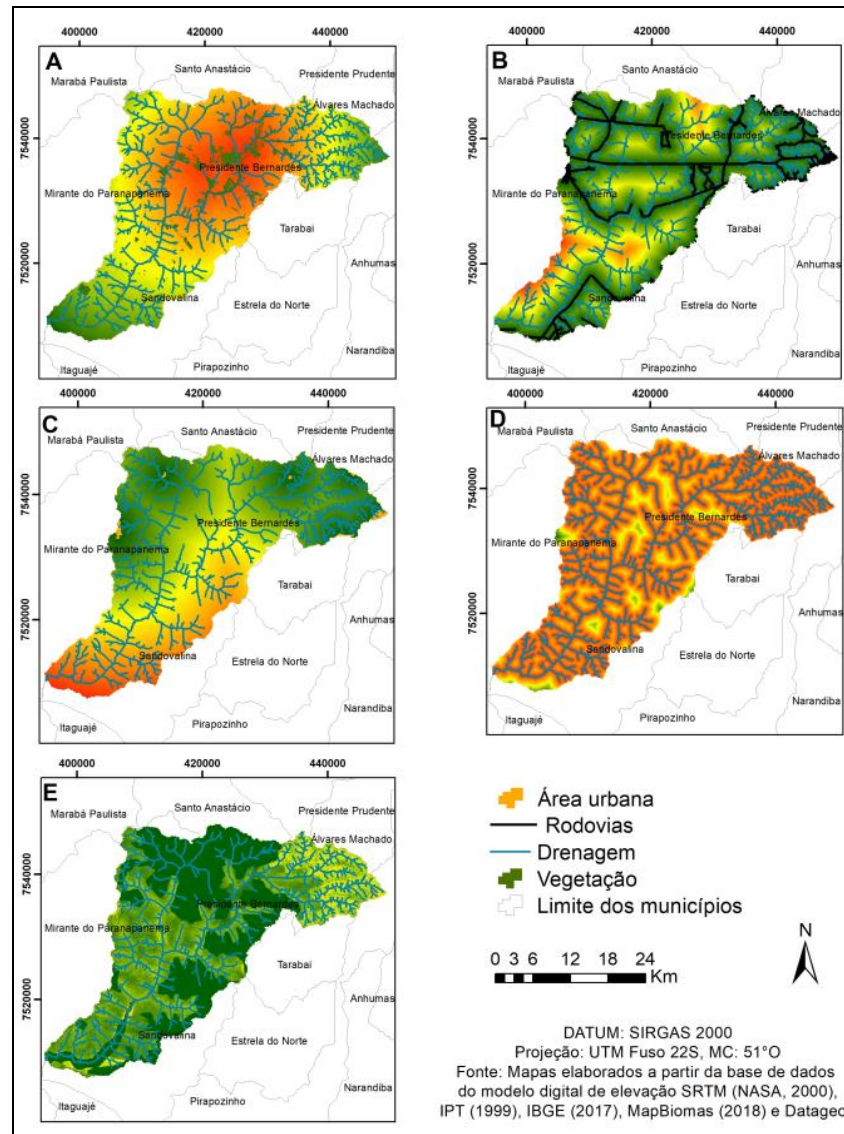

4. Área urbana

- Rodovias

- Drenagem

- Vegetação

Limite dos municípios
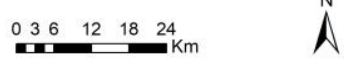

DATUM: SIRGAS 2000

Projeçẵo: UTM Fuso $22 \mathrm{~S}, \mathrm{MC}: 51^{\circ} \mathrm{O}$

Fonte: Mapas elaborados a partir da base de dados do modelo digital de elevaçăo SRTM (NASA, 2000), IPT (1999), IBGE (2017), MapBiomas (2018) e Datage

Fonte: Elaborado pelos autores. 
Para cada modelo de inferência aplicado geramos um mapa (Figura 4 e 5) indicativo das áreas prioritárias para reflorestar na bacia. As áreas próximas da cor vermelha demonstram que é maior prioridade de reflorestamento, já as áreas próximas da cor verde indicam menor prioridade. Por meio de uma análise visual, os produtos gerados demonstraram grandes variações entre os modelos de inferência (comparação entre Gamma 0,4 0,6 0,8 e CLP).

Figura 4 - Mapa de áreas prioritárias para reflorestamento na bacia do rio Pirapozinho obtido pelo método CLP.

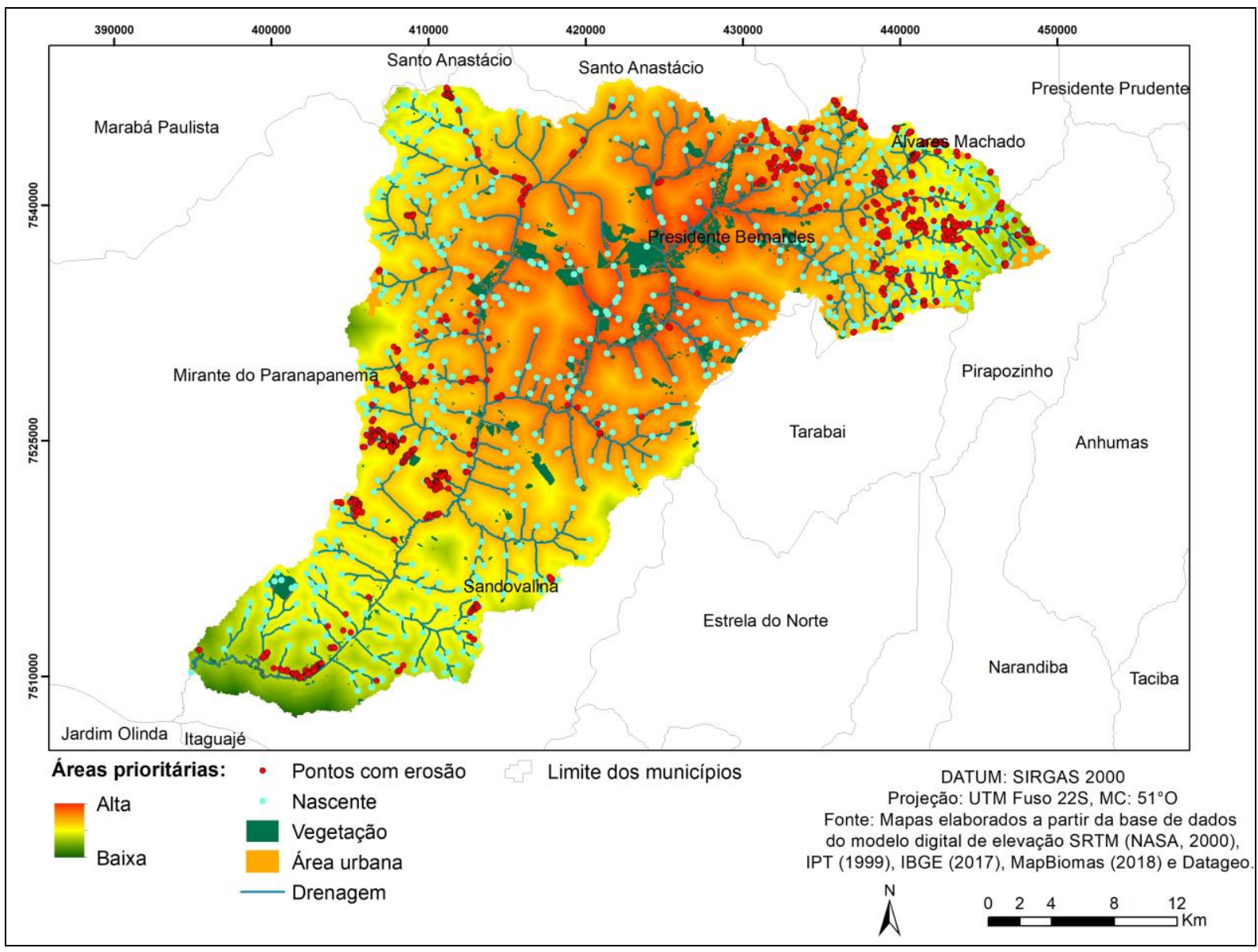

Fonte - Elaborado pelos autores.

Os pontos de erosão da figura 4 totalizam 951 pontos que correspondem a locais de erosão na área em estudo, os quais foram determinados pela fotointerpretação de Imagens do Google Earth Pro. Todos os pontos foram validados por modelos de regressão logística (MAFRA et al., 2020).

As áreas indicadas como prioritárias na Figura 4 possuem influência dos mapas de "distância de fragmentos de vegetação" e "distância de drenagem" (Figura 3). Notamos que a área central da bacia demonstrou ter uma alta prioridade e isso se deve a comparação pareada, pois o critério "fragmento de vegetação" teve o maior peso diante de todos os outros critérios adotados (Tabela 2). Para suas subclasses adotamos pesos de acordo com o tamanho da área de cada fragmento (ha), ou seja, quanto maior a área do fragmento, maior o peso adotado (Tabela 1). Vale ressaltar que a região central da bacia, onde demonstra alta prioridade (Figura 4), é composta pelos fragmentos com maior área (ha) de vegetação, como podemos observar na Figura 3(A). Observamos também que a áreas distantes dos grandes fragmentos também foram caracterizadas como prioritárias. Isso se deve as redes de drenagem, pois se encontram em toda a extensão da bacia, receberam o segundo maior peso por meio da comparação pareada, e, portanto, isso justifica as áreas indicadas como prioritárias na Figura 4. 
Assim como o CLP, o Gamma 0,8 (Figura 5-C) também priorizou as áreas consideradas mais importantes. No entanto, o Gamma 0,8 limitou melhor algumas áreas de priorização, e não necessariamente todo o canal da drenagem da bacia como o CLP. Por meio de análise visual, observamos que o Gamma 0,8 priorizou as áreas com maior vulnerabilidade à erosão na bacia em estudo (Figura 3-E), e não toda a sua extensão.

Figura 5 - Mapa de áreas prioritárias para reflorestamento na bacia do rio Pirapozinho obtido por diferentes operadores gamma. (A) Fuzzy por operador gamma com expoente 04; (B) Fuzzy por operador gamma com expoente 0,6; (C) Fuzzy por operador gamma com expoente 0,8.

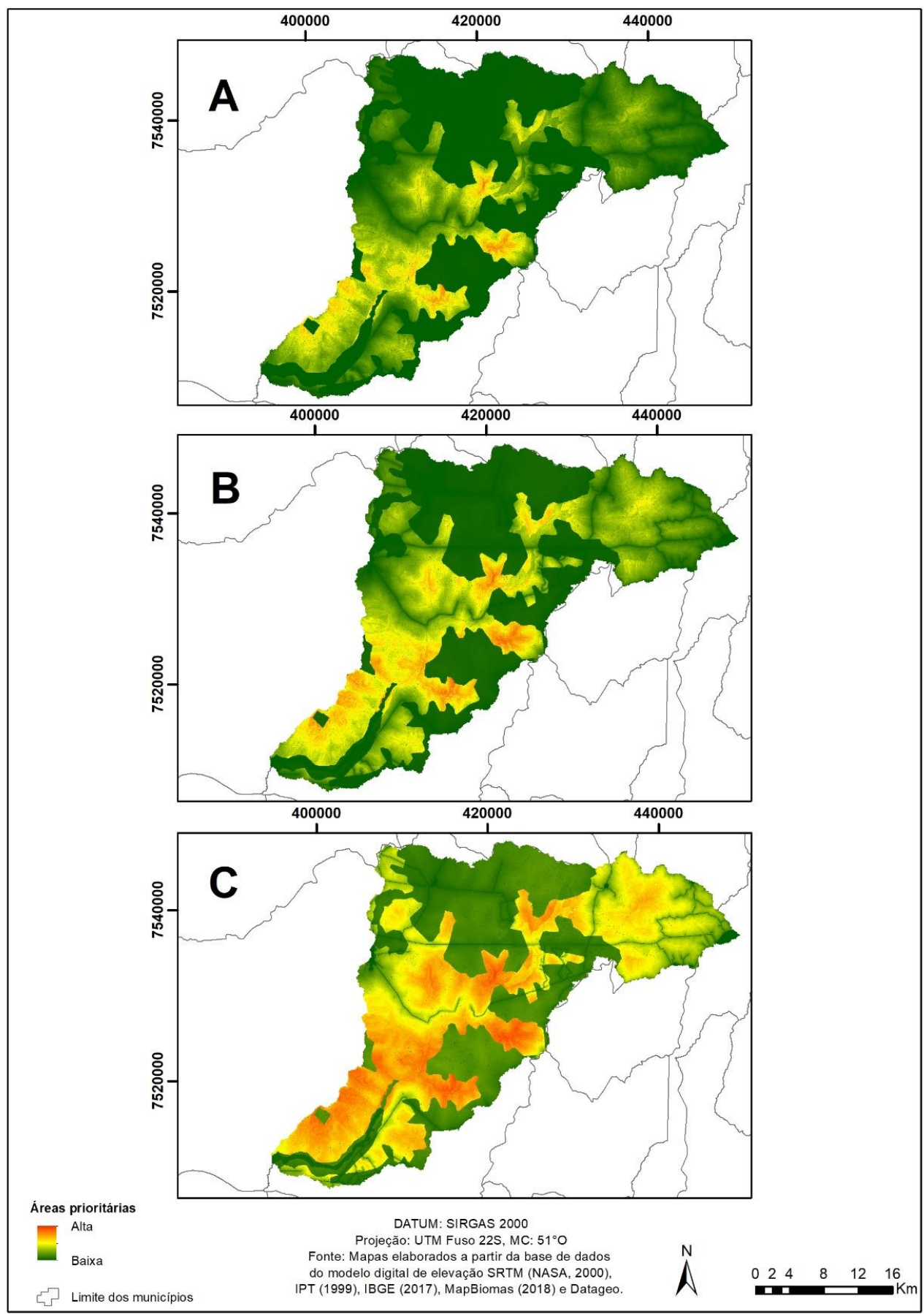

Fonte - Elaborado pelos autores. 
Por meio de análise visual, ao associar as áreas prioritárias encontradas nas Figuras 4 e 5 , observamos que o CLP e o Gamma 0,8 possibilitaram a identificação de áreas prioritárias para recuperação florestal. Incluímos os pontos de erosão, nascentes, vegetação, áreas urbanas nas figuras 4 e 6 para uma melhor explanação dos mapas temáticos. É importante que esses pontos estejam inclusos no planejamento de recuperação florestal de bacias, pois são fatores de extrema importância quando se pensa em conservação e preservação ambiental.

Figura 6 - Mapa de áreas prioritárias para reflorestamento na bacia do rio Pirapozinho obtido por diferentes operadores gamma.

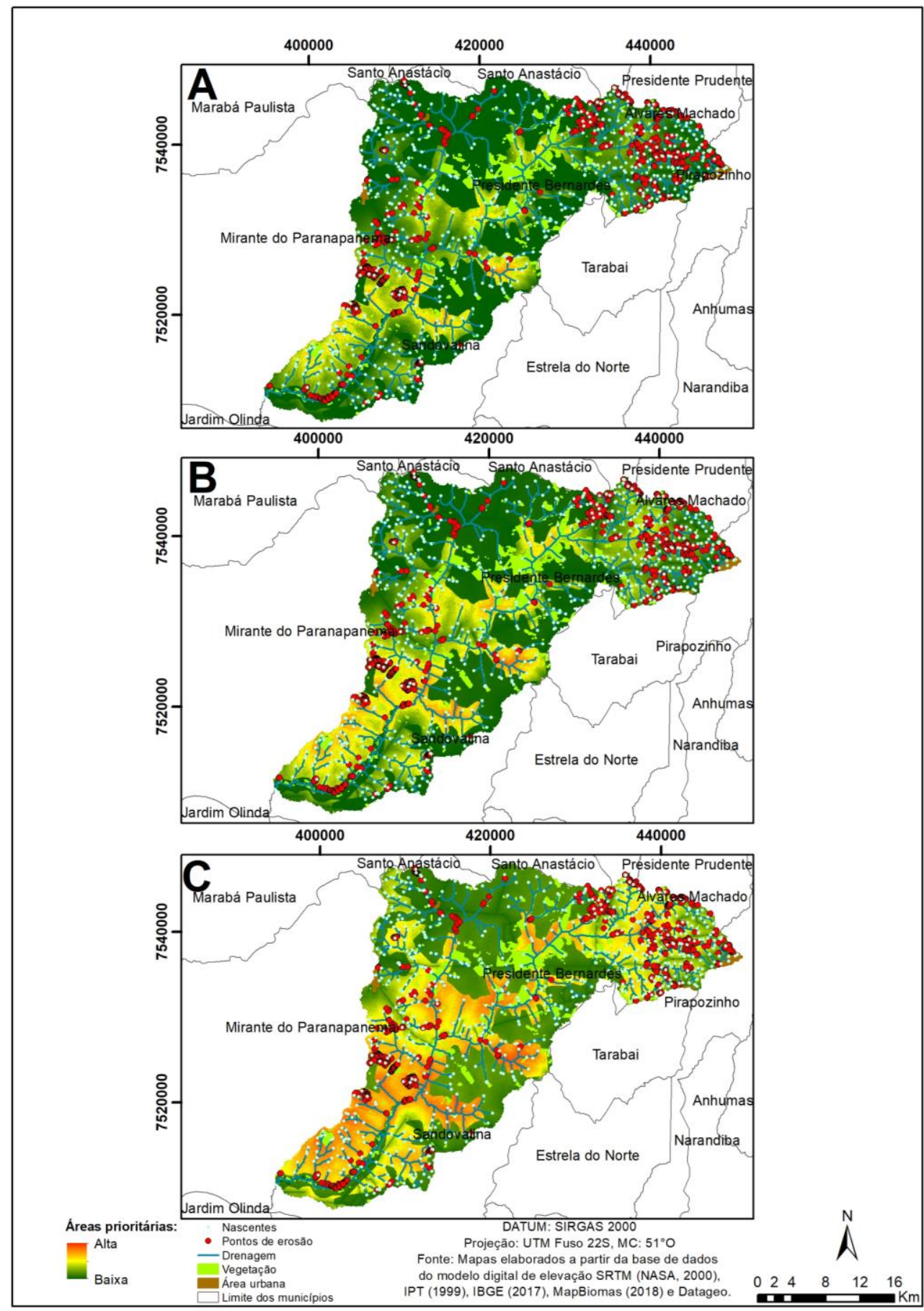


No entanto, como já citado, existem regiões da bacia onde há grande concentração de afluentes, e na Figura 4 como a drenagem teve o segundo maior peso na comparação pareada, consequentemente as áreas de nascentes, APP e os pontos de erosão foram priorizados. Vale salientar, que grande parte dos pontos de erosão estavam presentes em áreas próximas às redes de drenagem, portanto em áreas indicadas como prioritárias.

Visto que os métodos tiveram comportamentos diferentes, notamos que podemos abordar o trabalho em dois cenários: o primeiro com o CLP, onde podemos dar pesos aos critérios direcionando a ordem de importância de cada um deles que determinará as áreas prioritárias da bacia. Neste caso, priorizamos os grandes fragmentos de vegetação e a rede de drenagem. No segundo cenário observamos que podemos ter uma prioridade um pouco mais restrita para áreas que consideramos mais vulneráveis do que outras, ou seja, áreas de maior declividade e maior densidade de drenagem. A porção Noroeste da bacia do rio Pirapozinho no mapa de vulnerabilidade a erosão, apresentou-se alta densidade de drenagem associada com altos valores de declividade, e esses critérios foram os que obtiveram maior peso na ponderação pelo AHP (MAFRA et al., 2020). Diante disso, é importante observarmos que dependendo do tipo de planejamento ou implementação de projetos voltado a recuperação florestal, é significativo ter conhecimento das características da área e qual cenário desejará adotar.

\section{CONCLUSÃO}

Os métodos (CLP x FUZZY) apresentaram resultados distintos. A metodologia Fuzzy teve uma grande interferência do tema "vulnerabilidade à erosão", que teve comportamento diferente de acordo com o expoente do gamma. Grandes áreas classificadas como prioritárias pelo CLP, ficaram com baixa prioridade na metodologia Fuzzy. Constatamos que podemos abordar dois cenários neste trabalho: o primeiro com o método CLP onde priorizamos áreas com grandes fragmentos de vegetação e alta densidade de drenagem, e o segundo com o método Gamma, que priorizou áreas vulneráveis da bacia. O modelo de integração proposto satisfaz a identificação de áreas para a recuperação de florestas em bacias hidrográficas, e diferentes cenários podem ser construídos.

Recomendamos que trabalhos futuros utilizem outros métodos para a construção de novos cenários, com diferentes critérios e/ou abordem métodos que validem os cenários apresentados. Recomendamos também validações com idas a campo da área de estudo.

Para planejamentos futuros da bacia do rio Pirapozinho, caso a mesma seja considerada em um futuro projeto de recuperação de vegetação, recomendamos o mapa temático elaborado pelo método Fuzzy por operador gamma com expoente 0,8 , priorizando a região Oeste e Noroeste da bacia. Visando a conservação dos recursos hídricos, essas áreas da bacia têm grandes declividades, uma alta concentração de afluentes, consequentemente uma alta densidade de drenagem, portanto visando a conservação das áreas vulneráveis a erosão, nascentes, APPs e afluentes, a região Noroeste e região Oeste da bacia são as áreas prioritárias para se reflorestar.

\section{AGRADECIMENTO}

Agradecimento ao Núcleo de Estudos Ambientais e Geoprocessamento - NEAGEO da Universidade do Oeste Paulista - UNOESTE e a Coordenação de Aperfeiçoamento de Pessoal de Nível Superior (CAPES).

\section{REFERÊNCIAS}

ALMEIDA, F. C.; SILVEIRA, E. M. O.; PAIVA, L. L.; JUNIOR, F. W. A., 2019. Mapping of priority areas for forest recovery using multicriteria analysis in the brazilian atlantic forest. RAOEGA - O espaço geográfico em análise, v. 46, n. 03, p. 113-124. https://doi.org/10.5380/raega.v46i3.67075

ARANA, A. R. A.; ULIANA, M. R.; EVANGELISTA, C. R. L., 2019. Produtor familiar x Agronegócio canavieiro: impactos do cultivo da cana na produção do bicho da seda em Mirante do Paranapanema. Geosul, v. 34, n. 71, p. 623-639. https://doi.org/10.5007/1982-5153.2019v34n71p623

$\begin{array}{lllll}\text { Caminhos de Geografia } & \text { Uberlândia-MG } & \text { v. 21, n. } 77 & \text { Out/2020 } & \text { p. 220-233 Página } 230\end{array}$


BIANCHINI, C. D.; OLIVEIRA, G. G., 2019. Geoprocessamento aplicado à identificação de áreas aptas para a implantação de unidades de conservação no Vale do Taquari, RS. Revista Brasileira de Cartografia, v. 71, n. 2, p. 513-541. https://doi.org/10.14393/rbcv71n2-48357

BRANCALION, P. H. S.; GARCIA, L. C.; LOYOLA, R.; RODRIGUES, R. R.; PILLAR, V. D.; LEWINSOHN, T. M., 2016. Análise crítica da Lei de Proteção da Vegetação Nativa (2012), que substituiu o antigo Código Floresta: atualizações e ações em curso. Natureza \& Conservação 14. https://doi.org/10.1016/j.ncon.2016.03.004

BRASIL. Lei 12.651/2012. Disponível em: < http://www.planalto.gov.br/ccivil_03/_ato20112014/2012/lei/l12651.htm>. Acesso em 24 nov. 2019.

CÂMARA, G.; DAVIS, C.; MONTEIRO, A. M. V., 2001. Introdução à Ciência da Geoinformação. INPE, São José dos Campos - SP.

CATELANI, C. S.; BATISTA, G. T.; TARGA, M. S.; DIAS, N. W., 2012. Determinação de áreas prioritárias para o restabelecimento da cobertura florestal, apoiada no uso de geotecnologias. Revista Ambiente \& Água, Taubaté, v. 7, n. 3, p. 113-126. https://doi.org/10.4136/ambi-agua.964

DINIZ, A.; FURLAN, S. A., 1998. Relações entre as classificações fitogeográficas, fitossociologia, cartografia, escalas e modificações sócio-culturais no Parque Estadual de Campos de Jordão (SP). Revista do Departamento de Geografia, v. 12, p. 123-161. https://doi.org/10.7154/RDG.1998.0012.0006

FILOSO, S; BEZERRA, M. O.; WEISS, K. C.; PALMER, M. A., 2017. Impacts of forest restoration on water yield: A systematic review. Plos One, v. 12, n.8. https://doi.org/10.1371/journal.pone.0183210

FUSHIMI, M.; NUNES, J. O. R., 2018. Vulnerabilidade ambiental aos processos erosivos lineares das paisagens de parte dos municípios de Marabá Paulista (SP) e Presidente Epitácio (SP), Brasil. Revista da Associação Nacional de Pós-Graduação e Pesquisa em Geografia (Anpege) 14, p. 05-27. https://doi.org/10.5418/RA2018.1423.0001

FUSHIMI, M.; NUNES, J. O. R.; NAKAMURA, R. Y.; TAKATA, L. T. O., 2013. Vulnerabilidade ambiental e aplicação de técnicas de contenção aos processos erosivos lineares em áreas rurais no município de Presidente Prudente - SP. Revista Brasileira de Geomorfologia 14, p. 343-356. https://doi.org/10.20502/rbg.v14i4.435

GONÇALVES, S. R. A.; ARAÚJO, R. R.; IMAI, N. N., 2016. Mapeamento do grau de fragilidade com processo analítico hierárquico e operadores fuzzy gama na detecção áreas de fragilidade ambiental. Revista Brasileira de Cartografia 68, p. 327-337.

HARRIS, L.D., 1984. The fragmented forest: Island biogeography theory and the preservation of biotic diversity. Chicago: University of Chicago Press, p. 230. https://doi.org/10.7208/chicago/9780226219950.001.0001

HU, T.; CHANG, J.; LIU, X.; FENG, S., 2017. Integrated methods for determining restoration priorities of coal mining subsidence areas based on green infrastructure: -A case study in the Xuzhou urban area, of China. Ecological indicators. https://doi.org/10.1016/j.ecolind.2017.11.006

ITESP. Fundação Itesp: sua história e realizações, evolução das políticas agrárias e fundiária no Estado de São Paulo. São Paulo: Itesp, 2013, p. 304.

KANGAS, J.; STORE, R.; LESKINEN, P.; MEHTÄTALO, L., 2000. Improving the quality of landscape ecological forest planning by utilizing advanced decision-support tools. Forest Ecology and Management, Amsterdam, v.132, p.157-171. https://doi.org/10.1016/S0378-1127(99)00221-2

MAFRA, C. M.; PINHEIRO, M. M. F.; CICERELli, R. E.; OSCO, L. P.; ALVES, M. A.; RAMOS, A. P. M. Validação de mapa de vulnerabilidade a erosão por aprendizagem de máquina. Revista Brasileira de Geografia Física, v. 13, n. 02, p. 564-575, 2020. https://doi.org/10.26848/rbgf.v13.2.p564-575

MAGNANI, R.; LIMA, E. S. O impacto da nova estação de tratamento de efluentes do município de Resende-RJ para o fortalecimento do conceito de cidade sustentável. Revista de Direito e Sustentabilidade, Salvador, v.4, n. 1, p. $19-38$, jan/jun 2018. https://doi.org/10.26668/IndexLawJournals/2525-9687/2018.v4i1.4112

MALCZEWSKI, J., 2010 Multiple Criteria Decision Analysis and Geographic Information Systems. In: GRECO, S.; EHRGOTT, M.; Figueira, J. R. Multiple Criteria Decision Analysis, 02, 369-395. https://doi.org/10.1007/978-1-4419-5904-1 13

$\begin{array}{lllll}\text { Caminhos de Geografia } & \text { Uberlândia-MG } & \text { v. 21, n. } 77 & \text { Out/2020 } & \text { p. 220-233 Página } 231\end{array}$


MELLO, K.; COSTA, D. R.; VALENTE, R. A.; VETTORAZZI, C. A., 2018. Multicriteria Evaluation for Protected Area Definition Aiming at Water Quality Improvement. Floresta e Ambiente 25. https://doi.org/10.1590/2179-8087.013416

MOSTERT, E.; GAERTNER, M.; HOLMES, P. M.; O'FARRELL, P.; RICHARDSON, D. M. A multicriterion approach for prioritizing areas in urban cosystems for active restoration following invasive plant control. Environmental Management. Setembro, 2018. https://doi.org/10.1007/s00267-018$\underline{1103-9}$

NOSSACK, F. A.; ZIMBACK, C. R L.; SILVA, R. F. B.; SARTORI, A. A. C., 2014. Aplicação de análise multicriterial para determinação de áreas prioritárias à recomposição florestal. Irriga - Brazilian Jounal of irrigation and drainage, 19, 612-625. https://doi.org/10.15809/irriga.2014v19n4p612

NOSSACK, F. A.; ZIMBACK, C. R L.; SILVA, R. F. B.; SARTORI, A. A. C., 2014. Aplicação de análise multicriterial para determinação de áreas prioritárias à recomposição florestal. Irriga - Brazilian Jounal of irrigation and drainage, 19, 612-625. https://doi.org/10.15809/irriga.2014v19n4p612

OUYANG, W.; WU, Y.; HAO, Z.; ZHANG, Q.; BU, Q.; GAO, X., 2018. Combined impacts of land use and soil property changes on soil erosion in a mollisol area under long-term agricultural development 613-614, p. 798-809. https://doi.org/10.1016/j.scitotenv.2017.09.173

PINTO, L. V. A.; ROMA, T. N.; BALIEIRO, K. R. C., 2012. Avaliação qualitativa da água de nascentes com diferentes usos do solo em seu entorno. CERNE, v. 18, n. 3, p. 495-505. https://doi.org/10.1590/S0104-77602012000300018

POOR, E. E.; JATI, V. I. M.; IMRON, M. A.; KELLY, M. J., 2019. The road to deforestation: Edge effects in na endemic ecosystem in Sumatra, Indonesia. Plos One 14 (7). https://doi.org/10.1371/journal.pone.0217540

REZZADORI, T.; HARTMANN, M. T.; HARTMANN, P. A., 2016. Proximidade de rodovias pode influenciar a fragmentação florestal? Um estudo de caso no norte do Rio Grande do Sul. Biotemas, v. 29, n. 3, p. 21-28. https://doi.org/10.5007/2175-7925.2016v29n3p21

RODRIGUES, T.; TOMMASELLI, J. T. G.; ARROIO JUNIOR, P. P., 2018. Mapeamento da vulnerabilidade aos processos erosivos a partir de métodos multicriteriais: um estudo sobre as condições ambientais da bacia hidrográfica da represa da Laranja Doce, Martinópolis - SP. Revista Formação (Online), v. 25, n. 45, p. 67-91. https://doi.org/10.33081/formacao.v25i45.5310

ROSS, J. L. S., 1994. Análise empírica da fragilidade dos ambientes naturais e antropizados. Revista do departamento de Geografia, 08, p. 3-74. https://doi.org/10.7154/RDG.1994.0008.0006

SAATY, THOMAS L., 1990. How to make a decision: the analytic hierarchy process. European Journal of Operational Research, North-Holland. 48, p. 9-26. https://doi.org/10.1016/0377$\underline{2217(90) 90057-I}$

SARTORI, A. A. C.; SILVA, R. F. B.; ZIMBACK, C. R. L., 2012. Combinação linear ponderada na definição de áreas prioritárias à conectividade entre fragmentos florestais em ambiente SIG. Revista Árvore, Viçosa, v. 36, n. 6, p. 1079-1090. https://doi.org/10.1590/S0100-67622012000600009

SERPA, D.; NUNES, J. P.; SANTOS, J.; SAMPAIO, E.; JACINTO, R.; VEIGA, S.; LIMA, J. C.; MOREIRA, M.; CORTE-REAL, J.; KEISER, J. J.; ABRANTES, N., 2015. Impacts of climate and land use changes on the hydrological and erosion processes of two contrasting Mediterranean catchments. Science of the Total Environment 538, p. 64-77. https://doi.org/10.1016/j.scitotenv.2015.08.033

SOUTHWORTH, J., MUNROE, D., NAGENDRA, H., 2004. And cover change and landscape fragmentation- comparing the utility of continuous and discrete analyses for a western Honduras region. Agriculture, Ecosystems and Environment, v. 101, p. 185-205. https://doi.org/10.1016/. agee.2003.09.011

SUN, D.; ZHANG, W.; LIN, Y.; LIU, Z.; SHEN, W.; ZHOU, L.; RAO, X.; LIU, S.; CAI, X.; HE, D. FU, S., 2018. Soil erosion and water retention varies with plantation type and age. Forest Ecology and Management 422, p. 1-10. https://doi.org/10.1016/j.foreco.2018.03.048

TURNER, M. G., 1989. Landscape ecology: the effect of pattern on process. Annual Review of Ecology and Systematics, v. $20, \quad$ p. 171-197. https://doi.org/10.1146/annurev.es.20.110189.001131

$\begin{array}{lllll}\text { Caminhos de Geografia } & \text { Uberlândia-MG } & \text { v. 21, n. } 77 & \text { Out/2020 } & \text { p. 220-233 }\end{array}$ Página 232


URIBE, D.; GENELETTI, D.; CASTILLO, R. F.; ORSI, F., 2014. Integrating Stakeholder Preferences and GIS-Based Multicriteria Analysis to Identify Forest Landscape Restoration Priorities. Sustainability 6, p. 935-951. https://doi.org/10.3390/su6020935

VAlENTE, R. A.; PETEAN, F. C. S.; VETTORAZZI, C. A., 2017. Multicriteria decision analysis for prioritizing areas for forest restoration. CERNE 23, p. 53-60. https://doi.org/10.1590/01047760201723012258

VALENTE, R. O. A.; VETTORAZZI, C. A., 2005. Comparação entre métodos de avaliação multicriterial, em ambiente SIG, para a conservação e a preservação florestal. Scientia Forestalis 26 , 51-61.

VALENTE, R. O. A.; VETTORAZZI, C. A., 2008. Definition of priority areas for forest conservation through the ordered weighted averaging method. Forest Ecology and Management 256, 1408-1417. https://doi.org/10.1016/i.foreco.2008.07.006

Vettorazzi, C. A.; Valente, R. O. A., 2016. Priority areas for forest restoration aiming at the conservation of water resources. Ecological Engineering 94, 255-267. https://doi.org/10.1016/i.ecoleng.2016.05.069

VIANNA, V. M., TABANEZ, A. A. J \& BATISTA, J. L. F., 1997. Dinâmica e restauração de fragmentos na floresta atlântica. In: LAURANCE, W. F. \& BIERREGAARD, R. O. (Org.). Tropical Forest Remnants Ecology, Management and Conservation of Fragmented Communities. Chigaco and London: Univerty of Chicago Press, p. 351-365.

WU, L.; JIANG, J.; LI, G.; MA, X., 2018. Characteristics of pulsed runofferosion events under typical rainstorms in a small watershed on the Loess Plateau of China. Scientific Reports 8:3672. https://doi.org/10.1038/s41598-018-22045-x

Recebido em: 24/02/2020

Aceito para publicação em: 18/08/2020 